\title{
A Spatio-Temporal Modelling of Covid-19 Infections in Toronto's Neighbourhoods
}

\author{
Sze Hang (Hana) Fu $\mathbf{u}^{1,2}$ \\ ${ }^{1}$ Biostatistics Division, Dalla Lana School of Public Health, University of Toronto, Toronto, Canada \\ ${ }^{2}$ Centre for Global Health Research, St. Michael's Hospital, Unity Health Toronto, Toronto, Canada \\ E-mail: hana.fu@mail.utoronto.ca
}

Received May 15, 2020

Accepted for publication June 1, 2020

Published online September 5, 2021

\begin{abstract}
Introduction: Besides age and sex as established risk factors of COVID-19 infection, social factors are important determinants, with people of lower socioeconomic status suffer disproportionately from the disease. The city of Toronto has one of the highest COVID-19 infection rates in Canada. This analysis aims to explore the socioeconomic correlates associated with COVID-19 infection and the temporal trends among different age groups in Toronto using geospatial modeling.
\end{abstract}

Methods: A Bayesian spatio-temporal analysis was conducted using public COVID-19 cases data for Toronto. The case data were modeled using the Besag-York-Mollie (BYM) model, implemented in R-INLA. The model adjusted for age, sex, neighbourhood-level socioeconomic factors, crime rates, and population density. Random effects were included to account for neighbourhood-level variation and for spatial autocorrelation. Temporal trends of COVID-19 cases were modelled using second-order random walks to allow non-parametric estimations.

Results: The model estimates showed that men are at higher risk of COVID-19 infection. Among neighbourhood factors, higher home prices, education level, and population density are at lower risks, while belonging to an improvement area showed elevated risks. The temporal trends differed by age, with ages 20-59 showed increased risks over time, compared to the youngest and older age groups. Model predictions showed that northwest Toronto has higher risk compared to the rest of Toronto.

Conclusion: The higher COVID-19 infection risks in the Northwest will require increase public health effort to control disease spread in this area. The ecological correlates identified in this analysis will also help to guide the ongoing vaccination plans. 